\title{
Biofilme e feridas crônicas: reflexões para o cuidado de enfermagem
}

\section{Biofilm and chronic wound: reflections for nursing care}

\author{
Carlos Poblete Jara ${ }^{1}$ • Juliany Lino Gomes Silva ${ }^{2}$ Flávia Cristina Zanchetta ${ }^{3}$ - Thais Rojo ${ }^{4}$ Maria Helena Melo Lima ${ }^{5}$
}

\begin{abstract}
RESUMO
Objetiva-se refletir sobre o tema biofilme e ferida crônica para o cuidado de enfermagem. Estudo teórico-reflexivo, no qual os dados foram baseados em pesquisa na base de dados Scientific Electronic Library Online (SciELO) e PubMed, no período de 2010 à 2015, utilizando-se os descritores infecção, biofilmes e cicatrização de feridas. Os resultados apresentaram o crescimento microbiano em feridas crônicas é uma preocupação na prática clínica e a presença do biofilme prejudica o processo de cicatrização. 0 biofilme obtém nutrientes do plasma e do exsudato presentes no leito da ferida, e regula o metabolismo, a virulência e motilidade pela liberação e detecção de moléculas denominadas de quorum sensing. Abordagens no tratamento de feridas crônicas com foco no biofilme consistem na avaliação das características da ferida e na utilização de métodos de desbridamento para remoção da necrose e do esfacelo. Concluí-se que a limpeza do leito da ferida e o uso de antimicrobianos contribuem para o controle da carga microbiana, mas a administração destes produtos requer uma avaliação criteriosa. Novos métodos de diagnóstico para o controle do biofilme são necessários com vistas à prevenção, ao tratamento e cura das lesões em menor tempo.
\end{abstract}

Palavras-chave: Infecção; Biofilmes; Cicatrização de Feridas.

\section{ABSTRACT}

The aim is to reflect on biofilms in chronic wound for nursing care. A theoretical and reflective study based on Scientific Electronic Library Online (SciELO) and PubMed database, on the period 2010 to 2015. The used descriptors were infection, biofilms and wound healing. The results showed the microbial growth in chronic wounds is a concern in a clinical practice, and the presence of biofilm harms the healing process. The biofilm obtains plasma and exudate nutrients found in the wound bed, and regulates metabolism, virulence and motility by releasing and detecting molecules named quorum sensing. Approaches on treatment of chronic wounds focused on biofilm consist on the evaluation of the wound characteristics and on the usage of debridement methods to remove necrotic tissue and slough. It concludes that cleaning the wound bed help on controlling microbial load, but the usage of antimicrobial agents is also an expedient currently employed to control biofilm. The search for new diagnostic methods and biofilm control is necessary in order to prevent, to treat and to heal the wound in lesser time.

Keywords: Infection; Biofilms; Wound Healing.

${ }^{1}$ Mestre em Ciências da Saúde pela Faculdade de Enfermagem da Universidade Estadual de Campinas (UNICAMP) e Doutorando do Programa de Pós-Graduação da Faculdade de Enfermagem da Universidade Estadual de Campinas (UNICAMP).

${ }^{2}$ Mestre em Enfermagem - Doutoranda do Programa de Pós-Graduação da Faculdade de Enfermagem da Universidade Estadual de Campinas (UNICAMP) - e-mail: julianylg@hotmail.com ${ }_{3}^{3}$ Mestranda do Programa de Pós-Graduação da Faculdade de Enfermagem da Universidade Estadual de Campinas (UNICAMP) - e-mail: flaviac.zanchetta@gmail.com

${ }^{4}$ Enfermeira do Programa de Residência Multiprofissional da Faculdade de Enfermagem da Universidade Estadual de Campinas (UNICAMP) - e-mail: hojo.thais@yahoo.com

${ }^{5}$ Professora Doutora da Faculdade de Enfermagem da Universidade Estadual de Campinas (UNICAMP) - e-mail: mhmelolima@gmail.com

Os autores declaram não haver conflitos de interesse nem fontes de financiamento. 


\section{INTRODUÇÃO}

A função primária da pele é a proteção ao meio externo. Quando da presença de uma ferida, iniciase o reparo tecidual, um processo complexo e que envolve várias fases (proliferação celular, migração e remodelamento) com o objetivo de reestabelecer sua função primária ${ }^{1}$. A falha no processo de cicatrização de uma ferida pode prolongar o tempo de reparo e conduzir a complicações, tais como infecção, dor crônica, amputações, maiores taxas de mortalidade e aumento no tempo de internação ${ }^{1}$.

0 crescimento microbiano é mais evidente em feridas crônicas e compõe-se de espécies bacterianas que se aderem para a formação de microcolônias organizadas em comunidades, o que denominamos de biofilme ${ }^{2}$. Desenvolve-se em superfícies bióticas e abióticas, tais como os dispositivos médicos, fragmentos de tecido morto e dispositivos ortopédicos ${ }^{1-3}$.

Em feridas crônicas - como as úlceras neuropáticas, úlceras por pressão ou venosas -, acredita-se que o biofilme é responsável pelo atraso no processo de cicatrização. Essas bactérias representam uma verdadeira comunidade, com seus próprios sistemas de defesa, comunicação, sobrevivência e persistência ${ }^{3}$. Outra propriedade pertencente ao biofilme é sua capacidade de regular o metabolismo, a virulência e motilidade pela liberação e detecção de pequenas moléculas secretadas em seus arredores, denominadas de quorum sensing (QS) ${ }^{2}$.

Observou-se ao longo dos últimos anos que o biofilme em uma ferida crônica complexa mostra-se difícil de ser detectado e autores sugerem que a presença de esfacelo, necrose e substância viscosa com brilho na superfície da lesão representam biofilme $e^{4-5}$. Por outro lado, pesquisas realizadas da coleta de tecido do leito da ferida indicam que aparência visual para a detecção do biofilme não é um indicador conclusivo e que feridas com aparência saudável, quando avaliadas em laboratório, evidenciam presença de biofilme, contribuindo para retardo na cicatrização6 ${ }^{6}$.

Portanto, o objetivo deste estudo teórico-reflexivo é apresentar o tema biofilme e ferida crônica para o cuidado de enfermagem.

\section{MÉTODO}

Trata-se de um artigo de reflexão que utilizou como referencial teórico pesquisa realizada nas bases de dados SciELO (Scientific Electronic Library Online) e PubMed com os descritores infecção, biofilmes e cicatrização de feridas. Considerou-se artigos que proporcionaram bases teóricas de argumentação para o tema com relação ao impacto do biofilme na ferida e as evidências disponíveis para o seu controle e tratamento. Com o intuito de contemplar os princípios éticos este artigo considerou as Leis $n^{\circ} 9.610 / 98$ e $n^{\circ} 12.853 / 13$, que regulamentam os direitos autorais no Brasil.

\section{DISCUSSÃO}

\section{Impacto do biofilme na ferida}

Toda ferida aberta contém microrganismos de fonte endógena (da própria flora do paciente) ou exógena. $\mathrm{Na}$ fase inicial da formação da ferida crônica estes microrganismos são geralmente destruídos pelo sistema imune do hospedeiro, ação desempenhada principalmente pelos neutrófilos ${ }^{7}$.

No momento em que as bactérias planctônicas (que flutuam livres) entram em contato com uma superfície inicia-se o processo de estimulação à adesão irreversível e, em seguida, a produção de sinais para coordenação da diferenciação e formação de uma matriz de suporte. Diante disso ocorre o desenvolvimento de uma capa protetora composta por polissacarídeo e, ao mesmo tempo, a sua multiplicação em microcolônias que contêm, por sua vez, uma mistura de polissacarídeos, proteínas, ácido desoxirribonucléico (DNA), lipídios, fosfolipídios, carboidratos, sais minerais e vitaminas que cercam as colônias formadas por populações desenvolvidas a partir de uma única ou de múltiplas espécies ${ }^{8-9}$.

0 fenótipo biofilme permite proteger a bactéria do sistema de defesa do hospedeiro de antibióticos e agentes antimicrobianos, como a prata. Sabe-se que a presença de biofilme e de alta carga microbiana na ferida, interfere na fase inflamatória, com maior recrutamento de neutrófilos e macrófagos para o leito da ferida. Durante este processo, espécies bacterianas estimulam outros mecanismos moleculares que estabelecem um ambiente com alta atividade inflamatória, prejudicando as fases subsequentes o que favorece o atraso no processo de cicatrização e a manutenção da cronicidade da ferida ${ }^{10-11}$.

Fatores sistêmicos tais como doenças crônicas de base, insuficiência vasculares, desnutrição, malignidade e trauma de repetição quando controlados favorecem a cicatrização da ferida crônica ${ }^{12}$. A colonização bacteriana está relacionada aos fatores locais, estudo evidenciou que $93,5 \%$ de úlceras venosas apresentavam colonizadas com Staphylococcus aureus e 52,2\% com Pseudomonas aeruginosa $a^{12}$. Porém, feridas com presença de Pseudomonas apresentaram maior extensão e menor taxa de cicatrização. Isto pode ser explicado pela capacidade da $P$. aeruginosa eliminar os leucócitos polimorfonucleares (PMN), sendo provavelmente o principal fator no atraso da cicatrização de feridas crônicas colonizadas com esse patógeno ${ }^{13}$.

Pesquisa demonstrou que a presença de biofilme contribui para baixa tensão de oxigênio no leito da ferida, com a presença de micro elétrodos identificaram áreas de depleção de oxigênio dentro das colônias de biofilme. Áreas de anóxia podem explicar a presença de anaeróbios em espécies mistas de biofilmes ${ }^{14}$. Por outro lado, estudo recente demonstrou que a baixa tensão de oxigênio no leito da ferida contribui para cronicidade da ferida ${ }^{15}$. 
Existe esforço científico para elucidar o real papel do biofilme e a cronicidade da ferida, porém ainda existe um longo caminho de investigação. $O$ que está aceito é que a não cicatrização de feridas está relacionado a presença de biofilme. Por último, o conhecimento científico sobre este tema tem potencial para melhorar os resultados de saúde na prática clínica e avançar em tecnologias inovadoras no cuidado, sendo necessário maior investimento na translação do conhecimento das pesquisas em laboratório para as ações diárias no cuidado de feridas crônicas.

\section{0 controle do biofilme}

Novas abordagens de tratamento de feridas crônicas vêm ganhando destaque, como nos Cuidados de Feridas com Foco no Biofilme (CFFB) ou Biofilm-based wound care algorithm, o qual consiste na avaliação das características da ferida e na utilização de métodos de desbridamento para remoção da necrose e do esfacelo ${ }^{16}$.

A combinação entre a falta de uma resposta imune adequada, o retardo no reparo tecidual da ferida e a tolerância do biofilme a agentes antimicrobianos fazem com que as feridas crônicas tornem-se uma lesão com risco potencial de infecção?.

Feridas que não cicatrizam mesmo depois da aplicação de rigorosos padrões de cuidado devem chamar a atenção dos profissionais de saúde para um exame meticuloso à procura de sinais diretos ou indiretos de biofilme. Enquanto a umidade é essencial para a cicatrização de uma ferida, pouca umidade ou presença de grande quantidade de exsudato tornam o ambiente susceptível à formação de biofilme. Dessa forma, o controle de umidade é essencial para otimizar o leito da ferida para cicatrização e minimização da possibilidade de crescimento microbiano ${ }^{9}$.

Atualmente, o controle do biofilme pode ser realizado de diversas formas, por meio de substâncias com interferência na adesão e no QS, tratamento com fagoterapia, desbridamento e com a utilização de ultrassom de energia acústica de baixa freqüência ${ }^{17}$. Além destes, encontramos como tratamento a terapia por pressão subatmosférica, oxigênio hiperbárico, a bioengenharia de tecidos alternativos e a terapia tópica ${ }^{18-19}$.

Antimicrobianos tópicos como, por exemplo, agentes à base de iodo, clorexidine, Polihexametileno Biguanida (PHMB) e produtos à base de prata, são utilizados para a prevenção e tratamento de feridas ${ }^{19}$. Revisões sistemáticas demonstraram que o uso tópico do PHMB ou coberturas impregnadas com prata promovem um ambiente propicio ao processo de cicatrização, controle da dor e diminuição da carga microbiana. Porém, o número reduzido da amostra, falhas metodológicas, inconsistência na mensuração e o curto periodo de seguimento dos pacientes dificultam as evidências para prática clinica ${ }^{20-21}$. Sendo assim, são necessários mais estudos clínicos randomizados para investigar a efetividade dos antimicrobianos em diferentes tipos de feridas.
Qualquer produto selecionado deverá ser usado por um período contínuo e no mínimo de 7 a 10 dias, com reavalição antes de suspender ou continuar o tratamento ${ }^{22}$. Recente consenso recomenda a utilização de antimicrobiano em até 2 semanas, especialmente curativo à base de prata. Após 2 semanas é necessário avaliar a eficácia do tratamento por meio da melhora no reparo tecidual e busca por sinais de infecção ${ }^{23}$. Além disso, a ferida com suspeita de presença de biofilme deve ser desbridada e limpa regularmente, uma vez que ele possui capacidade de regenerar em poucos dias ${ }^{16}$.

Recentemente, as pesquisas desenvolvidas nesta área têm como objetivo identificar compostos não-nativos capazes de modular QS bacterianos ${ }^{8}$. Uma das estratégias mais estudadas diz respeito aos inibidores do quorum sensing (QSI), dentre estes as furanonas sintéticas. Esses compostos foram os primeiros que mostraram boa inibição do QS, mas cabe ressaltar também que dois QSI foram isolados a partir de fontes naturais, como o rábano (rabanete japonês) e o alho ${ }^{24-25}$.

Estudos em pacientes com feridas crônicas demonstram que os CFFB e a utilização de algoritmos para o auxílio na identificação dos biofilmes constituem estratégias de intervenção significativas na gestão do cuidado, visto que possibilitam a reversão do status de cronicidade das feridas e apresentam resultados concretos que estimulam as pesquisas neste campo. Em estudo realizado com 190 pacientes, obtevese $77 \%$ de cicatrização completa; destes, $47 \%$ eram pacientes com osteomielite e $69 \%$ com diabetes ${ }^{16-26}$.

A presença de uma ferida aberta é fonte potencial para a formação do biofilme, o que conduz ao retardo do processo de cicatrização.A compreensão deste fato na prática clínica irá contribuir para novos direcionamentos nas intervenções de enfermagem, principalmente no manejo da prevenção da formação do biofilme, sendo a primeira ação efetiva no tratamento das feridas crônicas.

\section{CONCLUSÃO}

O objetivo da limpeza do leito da ferida é remover os debris, reduzir a carga microbiana e ajudar no controle do biofilme. Porém, este procedimento nem sempre é suficiente, uma vez que o fenótipo biofilme age de forma resistente a diversos tipos de tratamento. Atualmente não existe evidência conclusiva quanto à eficácia dos antimicrobianos disponíveis no mercado para o controle do biofilme, prevenção da infecção e redução do tempo de cura da ferida.

No entanto, o biofilme tornou-se uma palavrachave entre a comunidade clínica e científica acerca da cicatrização de feridas crônicas, impulsionando o desenvolvimento de estratégias e tecnologias para a otimização do tratamento. Além disso, é fundamental que na prática clínica os profissionais sejam capazes de identificar quaisquer alterações no leito da ferida e propor novas abordagens com o intuito de otimizar o cuidado. 
Por outro lado, é necessário que as práticas de enfermagem no ensino estejam abertas para discussões relativas ao atual estado da arte da cicatrização de feridas crônicas, com ênfase nos guidelines clínicos e nas tecnologias envolvendo o tratamento destas feridas. As pesquisas são necessárias na busca de novos métodos de diagnóstico e de controle em modelos in vivo com o objetivo de permitir melhor gerenciamento e aperfeiçoamento da prática clínica.

\section{REFERÊNCIAS}

1. Demidova-Rice TN, Hamblin MR, Herman IM. Acute and impaired wound healing: pathophysiology and current methods for drug delivery, part 1: normal and chronic wounds: biology, causes, and approaches to care. Adv Skin Wound Care [Internet]. 2012 [acesso em 15 set 2016];25(7):30414. Disponível em: https://www.ncbi.nlm.nih.gov/ pubmed/22713781.

2. Percival SL, Hill KE, Williams DW, Hooper SJ, Thomas DW, Costerton JW. A review of the scientific evidence for biofilms in wounds. Wound Repair Regen [Internet]. 2012 [acesso em 13 ago 2016];20(5):647-57. Disponível em: https://www.ncbi. nlm.nih.gov/pubmed/22985037.

3. Harmsen M, Yang L, Pamp SJ, Tolker-Nielsen T. An update on Pseudomonas aeruginosa biofilm formation, tolerance, and dispersal. FEMS Immunol Med Microbiol [Internet]. 2010 [acesso em 02 set 2016];59(3):253-68. Disponível em: https:// www.ncbi.nlm.nih.gov/pubmed/20497222.

4. Metcalf DG, Bowler PG, Hurlow J. A clinical algorithm for wound biofilm identification. Acta Med Croatica [Internet]. 2016 [acesso em 02 fev 2017];70(1):73-9. Disponível em: https://www.ncbi.nlm.nih.gov/pubmed/24633059.

5. HurlowJ,Bowler PG.Clinical experience with wound biofilm and management: a case series. Ostomy Wound Manage [Internet]. 2009 [acesso em 10 ago 2016] ;55(4):38-49. Disponível em: https://www.ncbi.nlm.nih.gov/pubmed/19387095.

6. Fazli $M$, Bjarnsholt $T$, Kirketerp-Moller $K$, Jorgensen $B$, Andersen AS, Krogfelt KA, et al. Nonrandom distribution of Pseudomonas aeruginosa and Staphylococcus aureus in chronic wounds. J Clin Microbiol [Internet]. 2009 [acesso em 10 set 2016];47(12):4084-9. Disponível em: https://www.ncbi. nlm.nih.gov/pubmed/19812273.

7. Fazli $M$, Bjarnsholt $T$, Kirketerp-Møller $K$, Jørgensen $A$, Andersen CB, Givskov M, et al. Quantitative analysis of the cellular inflammatory response against biofilm bacteria in chronic wounds. Wound Repair Regen [Internet]. 2011 [acesso em 10 ago 2016] ;19(3):387-91. Disponível em: https://www. ncbi.nlm.nih.gov/pubmed/21518086.

8. Hansen MR, Le Quement ST, Jakobsen TH, Skovstrup S, Taboureau O, Tolker-Nielsen T, et al. Solid-phase synthesis and biological evaluation of $\mathrm{N}$-dipeptido L-homoserine lactones as quorum sensing activators. Chembiochem [Internet]. 2014 [acesso em 10 set 2016];15(3):460-5. Disponível em: https:// www.ncbi.nlm.nih.gov/pubmed/24436223.

9. Hurlow J, Couch K, Laforet K, Bolton L, Metcalf D, Bowler P. Clinical Biofilms: A Challenging Frontier in Wound Care. Adv Wound Care (New Rochelle) [Internet]. 2015[acesso em 10 ago 2016];4(5):295-301. Disponível em: https://www.ncbi. nlm.nih.gov/pmc/articles/PMC4432968/.

10. Phillips PL, Schultz GS. Molecular Mechanisms of Biofilm Infection: Biofilm Virulence Factors. Adv Wound Care (New Rochelle) [Internet]. 2012 [acesso em 06 fev 2017];1(3):10914. Disponível em: https://www.ncbi.nlm.nih.gov/ pubmed/24527289.

11. Zhao G, Usui ML, Lippman SI, James GA, Stewart PS, Fleckman P, et al. Biofilms and Inflammation in Chronic Wounds. Adv Wound Care (New Rochelle) [Internet]. 2013 [acesso em 06 fev 2017];2(7):389-99. Disponível em: https://www.ncbi.nlm. nih.gov/pubmed/24527355.

12. Gjodsbol K, Christensen JJ, Karlsmark T, Jorgensen B, Klein BM, Krogfelt KA. Multiple bacterial species reside in chronic wounds: a longitudinal study. Int Wound J [Internet]. 2006 [acesso em 15 fev 2017];3(3):225-31.Disponível em: https:// www.ncbi.nlm.nih.gov/pubmed/16984578.

13. Jensen P, Bjarnsholt T, Phipps R, Rasmussen TB, Calum $\mathrm{H}$, Christoffersen L, et al. Rapid necrotic killing of polymorphonuclear leukocytes is caused by quorum-sensingcontrolled production of rhamnolipid by Pseudomonas aeruginosa. Microbiology [Internet]. 2007 [acesso em 06 set 2016];153(Pt 5):1329-38. Disponível em: https://www.ncbi. nlm.nih.gov/pubmed/17464047.

14. Lawrence JR, Swerhone GD, Kuhlicke U, Neu TR. In situ evidence for microdomains in the polymer matrix of bacterial microcolonies. Can J Microbiol [Internet]. 2007 [acesso em 25 ago 2016];53(3):450-8. Disponível em: https://www.ncbi.nlm. nih.gov/pubmed/17538657.

15. James GA, Ge Zhao A, Usui M, Underwood RA, Nguyen $H$, Beyenal $\mathrm{H}$, et al. Microsensor and transcriptomic signatures of oxygen depletion in biofilms associated with chronic wounds. Wound Repair Regen [Internet]. 2016 [acesso em 15 set 2016];24(2):373-83. Disponível em: https://www.ncbi.nlm. nih.gov/pubmed/26748963.

16. Wolcott RD, Rhoads DD. A study of biofilm-based wound management in subjects with critical limb ischaemia.J Wound Care [Internet]. 2008 [acesso em 15 set 2016];17(4):145-8, 50-2, 54-5. Disponível em: https://www.ncbi.nlm.nih.gov/ pubmed/18494432.

17. Cooper RA, Bjarnsholt T, Alhede M. Biofilms in wounds: a review of present knowledge. J Wound Care [Internet]. 2014 [acesso em 15 set 2016];23(11):570, 2-4, 6-80. Disponível em: https://www.ncbi.nlm.nih.gov/pubmed/25375405.

18. Li T, Wang G, Yin P, Li Z, Zhang L, Liu J, et al. Effect of negative pressure on growth, secretion and biofilm formation of Staphylococcus aureus. Antonie Van Leeuwenhoek [Internet]. 2015[acesso em 12 ago 2016];108(4):907-17. Disponível em: https://www.ncbi.nlm.nih.gov/pubmed/26272011.

19. Boateng J, Catanzano O. Advanced Therapeutic Dressings for Effective Wound Healing - a review. J Pharm Sci [Internet]. 2015 [acesso em 10 set 2016];104(11):3653-80.Disponível em: https://www.ncbi.nlm.nih.gov/pubmed/26308473.

20. To E, Dyck R, Gerber S, Kadavil S, Woo KY. The Effectiveness of Topical Polyhexamethylene Biguanide (PHMB) Agents for the Treatment of Chronic Wounds: A Systematic Review. Surg Technol Int [Internet]. 2016 [acesso em $15 \mathrm{fev}$ 2017];45-51. Disponível em: https://www.ncbi.nlm.nih.gov/ pubmed/27608742. 
21. Lo SF, Hayter M, Chang CJ, Hu WY, Lee LL. A systematic review of silver-releasing dressings in the management of infected chronic wounds.J Clin Nurs [Internet]. 2008 [acesso em $10 \mathrm{fev}$ 2017];17(15):1973-85. Disponível em: https://www.ncbi.nlm. nih.gov/pubmed/18705778.

22. David K, Terry S, Keryln C, Jacqui F, Greg S, Joyce B. Ten Top Tips: Understanding and managing wound biofilm. Wounds International [Internet]. 2014 [acesso em 15 ago 2016];5(2):204. Disponível em: http://www.selwynfoundation.org.nz/ media/2029/identifying-infection-in-chronic-wound.pdf.

23. Keryln C, Jacqui F, David K, David L, Christina L, et al. International consensus. Appropriate use of silver dressings in wounds. An expert working group consensus. Wounds International [Internet]. 2012 [acesso em 12 fev 2017]:1-20. Disponível em: http://www.woundsinternational.com/media/ issues/567/files/content_10381.pdf.

24. Jakobsen TH, Bragason SK, Phipps RK, Christensen LD, van Gennip M, Alhede $M$, et al. Food as a source for quorum sensing inhibitors: iberin from horseradish revealed as a quorum sensing inhibitor of Pseudomonas aeruginosa. Appl Environ Microbiol [Internet]. 2012 [acesso em 15 ago 2016];78(7):2410-21. Disponível em: https://www.ncbi.nlm. nih.gov/pubmed/22286987.

25. Jakobsen TH, van Gennip M, Phipps RK, Shanmugham MS, Christensen LD, Alhede M, et al. Ajoene, a sulfur-rich molecule from garlic, inhibits genes controlled by quorum sensing. Antimicrob Agents Chemother [Internet]. 2012 [acesso em 15 ago 2016];56(5):2314-25. Disponível em: https://www.ncbi. nlm.nih.gov/pubmed/22314537.

26. Percival SL, Vuotto C, Donelli G, Lipsky BA. Biofilms and Wounds: An Identification Algorithm and Potential Treatment Options. Adv Wound Care (New Rochelle) [Internet]. 2015 [acesso em 15 ago 2016];4(7):389-97. Disponível em: https:// www.ncbi.nlm.nih.gov/pmc/articles/PMC4487216/. 